\title{
CONSTRUYENDO SENTIDO SOBRE INTERNET EN EL ESPACIO DE LA DIÁSPORA: MUJERES LATINAS INMIGRANTES EN GRANADA
}

\author{
CECILIA GORDANO \\ Universidad de Utrecht - Universidad de Granada
}

Recibido: 01/07/2009

Aceptado: 01/09/2009

\section{Introducción}

En los últimos años, varios enfoques académicos sobre Internet han experimentado un giro cualitativo y socioconstruccionista que problematiza los paradigmas tecnodeterministas y cuantitativos incapaces de dar cuenta de las complejidades y dinamismo de las sociedades contemporáneas ${ }^{1}$. Al nivel de usuari@s, esto adquiere especial importancia para los Estudios Feministas sobre Internet, al validar espacios de investigación que valoran las experiencias de mujeres particulares invisibilizadas en las abstracciones estadísticas ${ }^{2}$. El paradigma tecnodeterminista, cuyo principal slogan se reduce a que la tecnología conduce al cambio social ${ }^{3}$, considera la tecnología como aparatos, mecanismos y programas que impactan siempre positivamente en la sociedad, concibiendo ésta como un dominio separado e incontaminante. Este reduccionismo impide considerar

1. LieVrouw, Leah A. y LivingSTONE, Sonia M. «Introduction», en L.A. Lievrouw y S.M. Livingstone (eds). Handbook of New Media. Social Shaping and Consequences of ICTs. Londres, Sage, 2002, pp.1-16, p.3.

2. VAN ZOONEN, Liesbet, «Feminist Internet Studies». Feminist Media Studies, vol. $1, \mathrm{n}^{\mathrm{o}} 1$ (2001), pp. 67-72, p. 70. MAHAN, Amy. «ICT indicators for advocacy» en Global Information Society Watch 2007 Report. Montevideo, APC e IteM, 2007, pp. 77-87, p. 77.

3. LieVrouw, Leah A. y Livingstone, Sonia M., Op. cit., p. 3.

Feminismo/s 14, diciembre 2009, pp. 143-162 
los ricos entretejidos entre los medios tecnológicos, la acción humana y la estructura social (...), las actividades o prácticas de comunicación en la que nos involucramos para desarrollar y usar esos dispositivos; y los arreglos sociales $u$ organizacionales que se generan alrededor de los dispositivos y las prácticas ${ }^{4}$.

Esta crítica, popularizada por los enfoques que destacan la construcción social de la tecnología, es doblemente relevante. Primero, constituye un punto de partida para interpretar las implicancias sociopolíticas, económicas y culturales de la comunicación mediada por ordenador (CMC) a través de Internet en tiempos de globalización. En segundo lugar, habilita un marco interpretativo para considerar los artefactos tecnológicos como vehículos de sentido, modelos para «pensar con $»^{5}$, que adquieren un simbolismo que marca períodos históricos así como mecanismos de identificación ${ }^{6}$, según variables de género, edad, clase socioeconómica, nacionalidad, entre otros.

Cuando Internet trascendió las fronteras de sus orígenes militares en los laboratorios estadounidenses, primero a las universidades y luego al público en general, a principios de los años 90, el paradigma tecnodeterminista reverberó en una ola de ciberdiscursos optimistas que enmascararon la red de redes en un imaginario revolucionario y emancipador, prometiendo «superar las limitaciones del tiempo, el espacio o la corporalidad $»^{7}$. Su lógica trae reminiscencias de los grandes relatos de la modernidad y el progreso ${ }^{8}$, y resulta problemático al separar las Tecnologías de la Información y la Comunicación (TIC) del «panorama social que precede, da forma, contextualiza y continúa después de cualquier innovación tecnológica» .

4. Ibíd, p. 7.

5. Geertz citado en LIE, Merete «The new Amazons. Gender symbolism on the Net» in M. LIE (ed) He, She and IT Revisited: New Perspectives on Gender in the Information Society. Oslo, Gyllendal Norsk Forlag, 2003, pp. 251-276, p. 252.

6. LEANDER, Keavin. «Locating Latanya: The Situated Production of Identity Artifacts in Classroom Interaction» Research in the Teaching of English, vol. 37, $\mathrm{n}^{\circ} 2$ (2002), pp.198-250.

7. Eisenstein citada en PAASONEN, Susanna. «Free Tourists in the Land of Plenty? From cyberdiscourse towards politics of location», en Communication Front 2000 Book, Crossing Points East-West, 2000, <http://www.cfront.org/cf00book/en/susanna-locationen>, consultado el 05-03-2009.

8. Lievrouw, Leah A. «Determination and Contingency in New Media Development: Diffusion of Innovations and Social Shaping of Technology Perspectives» en L.A. LIEvRouw, y LivingStone, S. (eds.) Handbook of New Media. Social Shaping and Consequences of ICTs. Londres, Sage, 2002, pp. 183-200, p. 185.

9. LivingSTONE, Sonia M. «Part one: the changing social landscape. Introduction» en L. A. Lievrouw. y S.M. LivingStone. (eds.) Handbook of New Media. Social Shaping and Consequences of ICTs. Londres, Sage, 2002, pp. 17-21, p. 17. 
Atender a este panorama social exige un enfoque glocal ${ }^{10}$, que contextualice realidades concretas de grupos de personas específicas y sus relaciones con la tecnología en el escenario móvil de un mundo cada vez más interdependiente e interconectado. Un concepto clave para pensar la actualidad es el de flujos transnacionales: movimientos multidireccionales y continuos de capitales, información, imaginarios y personas que, con mayor o menor fortuna, atraviesan las fronteras geopolíticas y desdibujan los alcances y definiciones de los estados-nación ${ }^{11}$. El movimiento no es lo novedoso de este fenómeno; tampoco los sistemas de poder que habilitan o frenan unos y otros. Lo que históricamente marcaría un punto de inflexión es la velocidad, escala y volumen de circulación de estos flujos, sus articulaciones y desuniones, cómo ello repercute en diferentes regiones del globo simultáneamente ${ }^{12}$ y en las personas y grupos de modo diferencial.

En este escenario de continuidades y quiebres, abordaremos las intersecciones entre Internet, migración y diferencia sexual, por entender que abre espacios reflexivos donde confluyen viejos y nuevos paradigmas de desigualdad, cambio y resistencia. Así, este trabajo aspira a retratar cómo mujeres específicas se relacionan con Internet al nivel de usuarias en el marco de sus proyectos migratorios $^{13}$. El estudio se basa en una muestra compuesta por 14 mujeres de entre 30 y 60 años residentes en Granada, tomando como criterios de selección su origen latino y su vinculación a organizaciones de inmigrantes (la Asociación de Uruguayos y Uruguayas de Granada y la Asociación de mujeres inmigrantes uno=uno), así como su participación en cursos de informática ofrecidos en espacios para mujeres inmigrantes por la Concejalía de Igualdad de Oportunidades del Ayuntamiento de Granada y la Fundación Albihar. Con estas mujeres se exploraron los matices de sus vivencias como usuarias actuales de Internet, el anhelo de otras de serlo en un futuro cercano, sus expectativas, sus miedos, sus frustraciones y sus motivaciones. Las entrevistas tuvieron lugar entre febrero y abril de 2009, y apuntaron a responder a la pregunta central de esta investigación: ¿cómo las mujeres latinoamericanas inmigrantes residentes en Granada, dan sentido a la comunicación por ordenador al habitar el espacio de la diáspora? Su formulación se inspira en aquellos

10. RoberTSOn, Roland. Globalization. Social Theory and Global Culture. Londres, Sage, 1992.

11. Appadurai, Arjun. «Here and now» en N. Mirzoeff (ed) The Visual Culture Reader, Londres, Routledge, 2002. CASTELLS, Manuel. "Materials for an exploratory theory of the network society» British Journal of Sociology, vol. 51, $\mathrm{n}^{\circ} 1$ (2000), pp. 5-24.

12. Appadurai, Arjun. Op. cit.

13. Investigación realizada con el apoyo de la beca Erasmus Mundos para el Master en Estudios de las Mujeres y de Género en Europa (GEMMA).

Feminismo/s 14, diciembre 2009, pp. 143-162 
autores que apuestan por una "etnografía de los usos cotidianos de Internet» ${ }^{14} \mathrm{y}$ llaman la atención sobre la importancia de explorar las primeras experiencias de quienes se inician como usuari@s ${ }^{15}$. Mucho se ha escrito y se escribe sobre los beneficios (y también las desventajas) que las TIC representan para 1@s más desposeíd@s pero ¿qué esperan de y cómo vivencian las aludidas estos supuestos beneficios? ¿Qué las motiva a incursionar en Internet? ¿Es posible trazar similitudes en sus motivaciones, más allá de sus diferentes perfiles? En este sentido, resulta de especial interés lo que Avtar Brah llama el «espacio de la diáspora», que permite situar a las entrevistadas como habitantes de:

la interseccionalidad de diáspora, frontera y dis/locación como puntos de confluencia de los procesos económicos, políticos, culturales y psíquicos (...) donde múltiples posiciones subjetivas son yuxtapuestas, refutadas, proclamadas o desautorizadas; donde lo permitido y lo prohibido se interrogan eternamente; y donde lo aceptado y lo transgresivo se funden imperceptiblemente ${ }^{16}$.

El espacio de la diáspora permite, así, un análisis interseccional (o al decir de Brah «multiaxial») del cruce de fronteras no sólo territoriales, políticas y económicas, sino también culturales y psicológicas ${ }^{17}$. Son precisamente las dimensiones cultural y psicológica con respecto a Internet las que las mujeres entrevistadas caminan, de puntillas, a horcajadas, a prisa o con pausa según el caso, cuando se definen como usuarias de lo que por razones de edad, género, clase, formación y/o locación geopolítica, no coincidía con sus horizontes de expectativa.

Esta investigación implica aunar tradiciones teóricas, epistemológicas y metodológicas divergentes: los Estudios Culturales Feministas sobre Ciencia y Tecnología y los Estudios Postcoloniales. Los primeros surgen de la

14. SiLVERSTONE, Roger. Media and Technology in the Everyday Life of European Societies. Informe del European Media and Technology in Everyday Life Network, 2000-2003. London School of Economics and Political Science, 2003. <http://www.lse.ac.uk/collections/EMTEL/reports/silverstone_2003_emtel.pdf> consultado el 20-10-2009. BAKARDJIEVA, Maria. Internet society: The internet in everyday life. Londres, Sage, 2005.

15. VAN DijK, Jan y HACKER, Kenneth. "The Digital Divide as a Complex and Dynamic Phenomenon». The Information Society, 19 (2003), pp. 315-326. FrISSEN, Valerie. «The myth of the digital divide» en A. PICOT, J.C. BuRGELMAN y K. SCHRAPE. E-merging media: Communication and the media economy of the future, European Communication Council Report. Berlin, Springer, 2005, pp. 271-284. FRISSEN, Valerie. «The myth of the digital divide», en E-merging media: Communication and the media economy of the future, European Communication Council Report. Berlin, Springer, 2005, pp. 271-284.

16. BRAH, Avtar. «Diaspora, Border and Transnational Identities» en R. LEWIS y S. MiLLS (eds.) Feminist Postcolonial Theory: A Reader. Edimburgo, Edinburgh University Press, 2003, pp. 613-634, p. 631.

17. Ibíd., p. 209. 
intersección de tres áreas interdisciplinarias subversivas, tanto por su lugar marginal en el canon de las ciencias humanas y sociales, como por su tendencia a transgredir los límites disciplinarios de teorías, métodos y enfoques ${ }^{18}$ : los Estudios Culturales, los Estudios Feministas y los Estudios Sociales sobre Ciencia y Tecnología. Este espacio habilita un abordaje de género y tecnología como fenómenos culturales y relacionales ${ }^{19}$. Sus principios básicos son que la tecnociencia no es neutral sino profundamente marcada por el género, y que las mujeres no pueden ser esencializadas en una categoría homogénea, sino que «hay diferencias entre mujeres y en cada mujer ${ }^{20}$. En esta línea, género y tecnología son constructos culturales, dinámicos y performativos. El género es «un proceso y un verbo más que un sustantivo ${ }^{21}$, cuyo análisis debe considerar varios niveles de la vida social simultáneamente. En el mismo movimiento, podemos expandir la definición de tecnociencia de sustantivo a verbo, para una aproximación que destaque las prácticas y procesos cotidianos de sujetos encarnados y situados. Las teorías postconiales, por su parte, proveerán el marco específico para contextualizar los actuales flujos dispares de recursos y de personas, heredados de la época colonial, y la conceptualización de la otredad ${ }^{22}$.

Con estas herramientas, dedicaremos la primera sección a problematizar el concepto de brecha digital, al construir categorías de otredad con respecto a un usuario de Internet universal. En la siguiente sección, se propondrá una categoría analítica alternativa para habitar, la otredad digital táctica, que trasciende las taxonomías hegemónicas y reconoce un espectro de prácticas y experiencias situadas que se revelan mucho más ricas y diversas, y menos mensurables que el conocimiento obtenido a partir de los estudios cuantitativos.

18. LYKKE, Nina. «Feminist Cultural Studies of Technoscience and Other Cyborg Studies. A Cartography», en R. BRAIDOTTI et.al. The Making of European Women's Studies Volume IV, Utrecht: Athena, 2002, pp. 133-143.

19. HARAWAY, Donna.Testigo_modesto@segundo_milenio Hombrehembra_conoce Oncoratón. Nueva York, Routledge, 1997. GuRUMURTHY, Anita. Gender and ICT. Overview report. Institute of Development Studies, 2004 <http://www.bridge.ids.ac.uk/reports_gend_ CEP.html\#icts>, consultado el 19-05-2009. VEHVILÄINEN, Marja, «Understandings of Gender and Information Technology», en C. MÖRTBERG (ed.). Where do we go from here? Feminist Challenges to Information Technology, Suecia, Luleå University of Technology, 2000, pp.17-38.

20. VehviLÄInen, Marja, «Understandings of Gender ...». Op. cit., p. 21.

21. Ibíd., p. 22.

22. FERNÁNDEZ, Maria, «Postcolonial Media Theory», en Art Journal, vol. 58, no 3 (1999). DiRLIK, Arif, «The Global in the Local': The Postcolonial Aura. Third World Criticism in the Age of Global Capitalism». En A. McClintock y A. Mufti (eds.). Dangerous Liaisons. Gender, Nation and Postcolonial Perspectives. Minneapolis, University of Minnesota Press, 1997.

Feminismo/s 14, diciembre 2009, pp. 143-162 
Ello se ejemplifica en la penúltima sección, con los testimonios de las mujeres que colaboraron con esta investigación.

\section{La(s) brecha(s) digital(es)}

El énfasis cuantitativo de la Sociedad de la Información ${ }^{23}$ ha dado lugar a conceptualizar la brecha digital como una cartografía numérica basada en el grado de inclusión y exclusión que suponen las TIC para diferentes regiones, países y colectivos. Al principio se la definía simplemente en función del acceso, como la división entre conectados y no conectados. Si bien este enfoque está aún vigente, ha sido muy criticado por presentar una visión parcial y limitada de un fenómeno complejo.

Frissen advierte que se parte de premisas erradas, primero al asumir los efectos de las TIC como positivos en sí mismos y necesarios para todo ejercicio ciudadano. Ella desmitifica esta idea y defiende cómo 1@s no-usuari@s tienen «muy buenos motivos» para no incursionar en las TIC, como por ejemplo no sentirse aludidos por un diseño gestado desde el inicio para usuari@s avanzados, falta de motivación o el entorno particular de sus redes sociales. Otra premisa es la idea misma de brecha, que asume la diferencia como algo estático y sinónimo de desigualdad, e instaura la dicotomía de los que «tienen» o «ricos en información», y quienes «no tienen»o «pobres en información». Para Frissen las diferencias son dinámicas y varían, y muchos de quienes antes estaban ajenos a las TIC han empezado a interesarse a su manera. Además, no se puede limitar la explicación de las causas de exclusión a aspectos cognitivos (falta de habilidades y conocimientos) o falta de recursos financieros. Para Frissen, lo cultural pesa más en muchos $\operatorname{casos}^{24}$.

Las aproximaciones numéricas, además de confirmar la existencia de brechas digitales territoriales, económicas y generacionales, han constatado grosso modo la existencia de brechas digitales de género presentes en todas las demás. Ello se acentúa o disminuye según las regiones del mapa, y se hace especialmente crítica en los países del llamado tercer mundo. Sin embargo, al estudiar a las mujeres latinas inmigrantes en España, estas cifras pierden su correlato geopolítico y demandan nuevos enfoques y conceptualizaciones.

En los esfuerzos por hacer de la brecha digital un concepto realmente útil para aprehender la realidad, se pluralizó su misma definición, distinguiendo

23. WeBSTER, Frank. «The Information Society Revisited», en L. LieVROUw. y S. LivingSTONE (eds.). Handbook of New Media. Social Shaping and Consequences of ICTs. Londres, Sage, 2002, p. 22-33, p. 26.

24. FRISSEN, Valerie. Op. cit. 
al menos dos: una primera brecha digital concebida en función del acceso, y una segunda brecha digital en función de los usos. Centrar la atención en los usos permitió diferenciar la complejidad y diversidad de las aplicaciones cotidianas de 1@s usuari@s. Asimismo motivó exploraciones sobre tipos y dificultades de acceso a nivel psicológico y sociocultural, tales como temor, actitud negativa y falta de motivación, antes invisibilizadas ${ }^{25}$. Con estas nuevas formulaciones, varios estudios establecieron que «la gente mayor, los de nivel educativo bajo y una gran proporción de mujeres, y analfabetos (funcionales) están fuertemente sobrerrepresentados entre la gente que carece de motivación ${ }^{26}$. Así, mientras las estadísticas sobre acceso tienden a retratar un panorama optimista en donde hombres y mujeres comparten porcentajes similares de representatividad ${ }^{27}$, los estudios cualitativos revelan cómo algunas mujeres enfrentan dificultades específicas por su condición de género.

En un amplio estudio sobre la segunda brecha digital, centrada alrededor de los usos y habilidades, Castaño concluye que ésta afecta en gran medida a las mujeres, e identifica ciertas dificultades para su plena integración en la Sociedad de la Información, vinculadas a su "posición en el mercado de trabajo»y, en estrecha vinculación, «problemas culturales e institucionales» ${ }^{28}$. Siendo lo laboral un ámbito clave donde las personas se inician en informática e Internet, la menor participación de las mujeres en empleos cualificados y bien remunerados con respecto a los hombres constituye un handicap de motivación. Asimismo, el tiempo es un bien escaso para mujeres con múltiples jornadas en que se superponen responsabilidades laborales con familiares y dejan poco tiempo personal para navegar en Internet o aprender informática. Habría entonces un entramado de desigualdades intrínsecas a los sistemas de género que condicionan cómo ciertas mujeres «perciben una menor utilidad de Internet que los hombres $»^{29}$. Ello se evidencia en muchos de los testimonios de las mujeres que colaboraron en la presente investigación.

Considerar los movimientos migratorios en el marco de la Sociedad de la Información ha permitido abordar la brecha digital desde una problemática específica que sugeriría la existencia de una tercera brecha digital. Ésta sería definida por las inequidades existentes en el acceso, uso y apropiación de

25. VAn DiJK, Jan y HACKER, Kenneth, Op. cit. Breemen y Terstroot, citados en FrISSEN, Valerie, Op. cit., p. 276.

26. VAN Dijk, Jan y HaCKER, Kenneth. Op. cit.

27. ITU. «Female Internet users as \% of total Internet users». Market Information and Statistics Unit. International Communication Union, 2006, <http://www.itu.int/ITU-D/ ict/statistics/at_glance/f_inet. html>, accedido el 28-07-2009.

28. Castaño, Cecilia (ed.). La segunda brecha digital. Madrid, Cátedra, 2008, p. 58.

29. Ibíd., p. 60.

Feminismo/s 14, diciembre 2009, pp. 143-162 
Internet por parte de cierto perfil de inmigrantes ${ }^{30}$ sin alfabetización digital, cuyos proyectos migratorios los llevan a países en los que los gobiernos han adoptado mecanismos de interacción y construcción de ciudadanía basados en Internet.

Los enfoques mencionados complejizan las tendencias meramente cuantitativas de los indicadores utilizados en los estudios sobre brecha digital. En general, dichos estudios suelen atender a la infraestructura, acceso y conectividad $^{31}$, y la mayoría de las veces, son realizados por entes reguladores o empresas de telecomunicaciones o marketing, con enfoques técnicos o empresariales antes que socioculturales. Es hora de cuestionar esa tendencia histórica de los indicadores TIC y, después de preguntarnos « ¿qué se está midiendo exactamente?», pasar a la cuestión fundamental de la que se hace eco este trabajo, es decir, «¿qué es lo que no se está midiendo?» ${ }^{32}$.

La utilidad del concepto de brecha digital estaría dada por su capacidad para visibilizar las desigualdades estructurales que reproducen los mecanismos de exclusión, matizando las promesas optimistas de los ciberdiscursos. Sin embargo, reinstaura las desigualdades que nombra al construir un factor más de otredad, midiendo accesos y usos en función de modelos universales y generalizadores que ocultan las diferencias dinámicas de grupos de usuari@s.

\section{Internet y los discursos de la otredad}

Los mecanismos de otredad activados en las conceptualizaciones sobre Internet van desde la victimización de los otros no conectados, hasta el ensalzamiento de las posibilidades emancipadoras que las TIC, en especial Internet, tiene para ell@s. Estos enfoques son correlatos de varias posiciones más o menos polarizadas: quienes conceptualizan Internet como un medio horizontal, participativo y desjerarquizado, a través del cual los sujetos otros tienen

30. Morales, Juan J. y RodríGUEZ, $\mathrm{M}^{\mathrm{a}}$ del Carmen. «Inmigración y ciudad digital: reflexiones entorno a la tercera brecha digital», Archivo del Observatorio para la CiberSociedad, 2008. <http://www.cibersociedad.net/archivo/articulo.php?art=232> consultado el 05-03-2009. Mosco, Vincent, The Digital Sublime. Myth, Power, and Cyberspace, Massachusetts, MIT Press, 2004, p. 17-49.

31. MAHAN, Amy. Op. cit., p.77.

32. PISCITELLI, Alejandro. «Inmigrantes digitales vs. nativos digitales. La migración digital, un concepto bastante ambicioso, sin fecha <http://portal.educ.ar/debates/educacionytic/nuevos-alfabetismos/inmigrantes-digitales-vs-nativos-digitales.php $>$ consultado el 22-06-2009. 
grandes posibilidades de cambiar su posición de subordinación ${ }^{33}$ (por ejemplo, para fortalecer las frágiles ciudadanías de las personas inmigrantes $\left.{ }^{34}\right)$; y quienes ven Internet con mayor cautela, como otro motivo de opresión y desigualdad ${ }^{35}$, sustrayendo toda o parte de agencia a los más desposeídos.

Las mujeres latinas inmigrantes constituyen un colectivo marcado triplemente por la otredad en el entendimiento binario de la realidad social que jerarquiza las diferencias entre hombres y mujeres, primer y tercer mundo, nacionales e inmigrantes. Muchas de ellas están, además, en la intersección de las variantes de brechas digitales antes mencionadas. Los mecanismos que otrorizan a las mujeres es la invisibilización de su existencia y de sus experiencias con/en Internet. En este artículo se busca revertir, al menos parcialmente, esta invisibilización, a la vez que se apuesta por una forma productiva y afirmativa de entender la otredad.

Por un lado, el concepto de «otredad inapropiada/ble» que Haraway ${ }^{36}$ retoma de Trinh Minh-ha, es ilustrativo para moldear la conceptualización de la otredad que aquí se desarrolla. Lo inapropiable estaría dado por la imposibilidad de ser clasificado como lo Uno ni como lo Otro, ante el rechazo de los lugares asignados por las narrativas occidentales modernas de las políticas de identidad: «lo inapropiado es una forma de figurar la diferencia como una diferencia critica desde/hacia dentro» extensible a las relaciones entre humanos, lo orgánico y lo tecnológico no humano ${ }^{37}$.

A su vez, podemos emplear el término otro digital para referirnos a grupos y personas subordinados en relaciones de poder cuya otredad encarnada reverbera en el acceso y la apropiación de las TIC. Esta otredad encarnada se manifiesta en aquellos cuerpos que no responden al modelo universal hu-

33. POSTER, Mark. What's the matter with the Internet?. Minneapolis, University of Minnesota Press, 2001, pp. 171-188; CASTELLS, Manuel. «Materials for an exploratory theory of the network society» British Journal of Sociology, 51(1), 2000, p. 5-24.

34. Mitra, Ananda. "Marginal Voices in Cyberspace». New Media Society, vol. 3, $\mathrm{n}^{\circ} 1$ (2001), pp. 29-48; MitRA, Ananda. «Theorizing Cyberspace: the idea of voice applied to the Internet discourse». New Media Society, vol 4, no 4 (2005), pp. 479-498; MARTínez, Manuel, Tucho, Fernando y García de Madariaga, José Mª. «Democracia Digital: Nuevos Medios y Participación Ciudadana. Experiencias en la Red de la Población Inmigrante en España». Portuaria, vol. 5, no 2 (2005), pp. 21-34; Hiller, Harry H. y Franz, Tara M. «New ties, old ties and lost ties: the use of the internet in diaspora». New Media and Society, vol. 6, nº 6 (2004), pp. 731-752.

35. Morales, Juan J. y RodríGUEZ, $\mathrm{M}^{a}$ del Carmen. Op. cit.; LEDWITH, Valerie et al. «Immigration, the internet, and spaces of politics». Political Geography, nº 21 (2002), pp. 989-1012.

36. HARAWAY, Donna. "The Promises of Monsters: A Regenerative Politics for Inappropriate/d Others». En L. Grossberg, C. Nelson y P. A. Treichler (ed.). Cultural Studies. Nueva York, Routledge, 1992, p. 295-337, p. 300.

37. Haraway, Donna. The Haraway Reader. Nueva York, Routledge, 2004, p.70.

Feminismo/s 14, diciembre 2009, pp. 143-162 
manista del hombre-blanco-joven-occidental-de clase media o alta, y que no gozan de los recursos materiales ni simbólicos para ocupar el espacio digital con esa lógica dominante. Este modelo universal ha caracterizado el perfil de los primeros usuarios de Internet, principalmente científicos, académicos y hobbistas $^{38}$, que aún hoy predominan como los expertos, tanto en el imaginario cultural como en lugares claves de poder en el diseño, desarrollo y toma de decisión sobre las TIC. Propongo, entonces, hibridizar la «otredad inapropiada/ ble» con la otredad digital para conceptualizar la otredad digital táctica. Esta otredad se inscribe en un espacio ambiguo, de límites indefinidos e indefinibles, plagado de contradicciones y grietas, en el cual los sujetos negocian sus condiciones de otredad y apelan a diversas tácticas para conseguir sus objetivos en las superposiciones e intersecciones de varios sistemas de organización y jerarquización de las diferencias en las sociedades contemporáneas. Para ejemplificarla, se atiende, en el siguiente apartado, a las palabras de las mujeres entrevistadas, cuyos testimonios nos recuerdan que hay diferentes formas de pensar, experimentar y habitar Internet, al margen de los discursos tecnodeterministas, mercantilistas, patriarcales, cyberoptimistas e incluso académicos.

\section{Las micropolíticas de Internet en la vida de las mujeres}

En esta sección se presenta una selección de los testimonios de las mujeres entrevistadas $^{39}$. M uchas de ellas trabajan como empleadas domésticas y fueron contactadas a partir de su participación en los cursos «Internet para tod@s» que, desde 2004, organiza la Concejalía de Igualdad de Oportunidades del Ayuntamiento de la ciudad de Granada. Este programa de alfabetización digital se enmarca en el Plan Municipal de Igualdad de Oportunidades entre mujeres y hombres que actualmente va en su cuarta edición. Los cursos se ofrecen gratuitamente en el Centro Europeo de las Mujeres Mariana de Pineda a todas las mujeres residentes en Granada, en sesiones diarias de una hora y media durante dos semanas. Se trata de una estrategia política de acción afirmativa para motivar curiosidad y entusiasmo a las participantes. Vehviläinen destaca la importancia de este tipo de iniciativas orientadas a:

quienes se desvían del ideal (liberal), en una sociedad occidental de la información, del hombre blanco, de clase media y con estudios. Personajes diferentes y contradictorios, como la mujer que no puede tipear, deberían

38. BAKARDJIEVA, Maria. Op. cit., p. 4.

39. A los efectos de la presentación de los testimonios, se utilizan nombres ficticios. 
encontrar sitio en los cursos que se desarrollan en términos de «acceso equitativo» ${ }^{40}$.

En los testimonios es posible identificar inquietudes y valoraciones similares con respecto a la posición subjetiva de ser mujeres, inmigrantes y usuarias (potenciales y reales) de Internet, que trascienden las diferencias de sus contextos de enunciación. Así, todas las entrevistadas, independientemente de su perfil (aunque con distintos matices), valoraron positiva y necesaria la alfabetización digital. A continuación se propone un ordenamiento de sus narraciones en base a tres focos: las experiencias de (re)aprendizaje informático, la articulación de los roles maternales con los de usuarias de Internet, y la negociación del tiempo familiar y el tiempo propio.

\subsection{Aprender y recordar}

Nadia, 64 años, educadora social, se definió como «una analfabeta de Internet y de ordenadores», a pesar de haber aprendido diez años atrás y recurrir a ello diariamente en su trabajo. «Soy usuaria amateur, obligada, y siento que me domina el ordenador, que surgen cosas que no sé resolver». Esta percepción coincidiría con los resultados de algunos estudios que han indagado cómo las mujeres tienden a infravalorar el conocimiento tecnológico que poseen ${ }^{41}$.

Como se mencionara en la sección anterior, los ambientes laborales y educativos actúan como incentivo para la formación en TIC. En el caso de Nadia, lo laboral más que un incentivo fue sentido como un imperativo: «utilizo Internet por obligación, porque en el trabajo hay ordenadores, y hay que meterse para buscar datos». Sus inicios informáticos fueron autodidactas, sin cursos formales y con el apoyo de «compañeros más jóvenes que sabían más». Nadia se siente ajena a Internet y a la tecnología en general: «yo me resistía, el ratón me daba miedo, me parecía que iba a tocarlo y se iba a desarmar el ordenador. Sentía eso porque no lo había usado nunca». Este sentir lo atribuye a un tema generacional ya que, para ella, no afecta a los niños y las niñas que crecen con Internet. Su reticencia a convertirse en internauta no le impidió, sin embargo, entusiasmar a Regina, una amiga uruguaya, para que abriera su propio correo electrónico. Hoy, con 59 años, trabajadora social, Regina se define como una adicta a Internet y negocia los tiempos de uso del ordenador

40. VehVilÄInEN, Marja. «Gendered agency in Information Society: on located politics of technology». En S. PAASONEN y M. CONSALVO (eds.). Women and Everyday Uses of the Internet: Agency and Identity. Nueva York, Peter Lang Publishing, 2002, pp. 274-288, p. 288.

41. Castaño, Cecilia, Op. cit.; Henwood citada en Vehviläinen, Marja. «Gendered agency...» Op. cit., p. 275.

Feminismo/s 14, diciembre 2009, pp. 143-162 
doméstico con su marido e hijo. Aprender a manejar el ordenador e Internet fue para ella un logro personal de autonomía:

Yo quiero conseguir las cosas por mí misma, ser capaz, y no me gusta depender. (...) Siempre decía ¡tengo que conseguirlo! No tenía ordenador ni nada, fui a la Biblioteca de Andalucía, ahí te permitían conectarte a Internet durante una hora, pedí mi turno pero me pasé 50 minutos buscando la arroba, ¡no la encontraba por ningún sitio!

La tenacidad individual se complementó con cursos de capacitación en el trabajo:

Eso me ayudó a ir perdiendo miedos (...) El ordenador no ordena por sí [sólo], sino que es un mecanismo que uno [sic] tiene que saber cómo funciona y si no lo usas adecuadamente...Se me han borrado cosas por no grabarlas (...) Te tienes que equivocar un montón de veces para entender. Aparte soy lenta para escribir.

Al igual que Nadia, Regina atribuyó las dificultades y los miedos a una cuestión generacional, de no haber crecido con esa tecnología y sentirse «muy torpe para aprender». Sin embargo, su marido, de su misma generación, no expresó dificultades ni resistencias a aprender en su entorno laboral, ayudado por compañeros de trabajo. Para Regina, el apoyo y la paciencia de su marido y su hijo fue y es muy importante. «Al principio dependía mucho de ellos (...) mi marido me explicó 25.800 veces las cosas que me costaban». Y por supuesto el apoyo de Nadia, con la que empezó a intercambiar correos electrónicos. A su vez, ella misma motivó a dos amigas a incorporarse a Internet. Una de ellas se había divorciado y todavía mantenía la cuenta con el apellido del marido. «¡Dejate de joder! le dije, ¿cómo es posible? (...) Tanto la jorobé que un día me senté con ella y estuve como dos horas [creando su e-mail]». En un futuro cercano, Regina espera poder hacer un curso específico de Internet. «Lo que he aprendido es por el apoyo que he tenido, por mi interés, por mi motivación y por mis ganas».

En casa de Mirta, brasileña de 51 años, hay un ordenador de su hija con conexión a Internet pero ella no lo usa mucho. «Lo prendo pero sólo puedo escribir practicando las letras, escribo todo el nombre de las personas (...) Mi hija no tiene paciencia para enseñarme, y me dice que no entre». Ha empezado con entusiasmo los cursos del Ayuntamiento; quiere comunicarse con sus amigas a la distancia y preparar su Curriculum Vitae. «Lo más difícil es recordar... dónde hay que pinchar, eso me cuesta un poco». Por ahora, su acceso a Internet se restringe a las horas del curso, ya que no tuvo buena experiencia en el locutorio: 
Me da vergüenza pedir ayuda. El otro día pedí a la chica si podía poner mi fotografía en el CV, y ella me dice nosotros no estamos aquí para enseñar y yo le dije pero voy a pagar por la tarea, y al final otro muchacho de ahí me ayudó.

Por su parte, las mujeres más jóvenes buscan repasar y recordar los conocimientos ya adquiridos en sus países de origen. Úrsula, boliviana de 32 años, quiere refrescar lo que aprendió hace diez años mientras cursaba Comunicación Social y Secretaría Ejecutiva. «Se me ha olvidado un poco y he venido a recordar, porque si no practicas, se te va».

En Paraguay, su país natal, Diana, de 42 años, siempre trabajó como pequeña comerciante, hasta que un robo la fundió y motivó a viajar a Granada para trabajar y pagar sus deudas. Hace seis años que viajó sola, y luego trajo a su marido e hija. Ahora que está en el paro, aprovecha para mejorar lo que es su vocación: el comercio.

Cada día va más avanzado para estudios y negocio en formato electrónico. Tengo ordenador en casa pero no lo puedo utilizar porque no sé, quiero comprar una cosa y no puedo hacerlo por Internet. Y si pienso algún día tener un negocio y tengo que usar un ordenador, ¿cómo lo hago? Tengo que hacer un curso para aprender a usar el aparato.

Tiene una casilla de correo que le abrió su hija y así se comunica con sus hermanas que están en Buenos Aires. Desde que empezó el curso, ella también enseña a su hija cosas que la niña aún no sabe, y a su marido. «Ahora voy y doy clase en mi casa», dijo orgullosa.

\subsection{Las mamás conectadas}

La informatización como habilidad complementaria del rol maternal emergió en varios testimonios. Úrsula, cuyo proyecto migratorio consiste en regresar a Bolivia y reencontrarse con sus hijos, lo expresa de la siguiente forma:

Más que todo para guiarlos a mis hijos, porque si algún día me preguntan algo yo voy a estar ignorante en ese tema, y no quiero pasar vergüenza con mis hijos. Porque yo salí bachiller, estudié Comunicación Social, y que no sepa nada de ordenadores, ¡eso va a ser la leche!

Para Rosario, colombiana de 43 años, también es importante ser una madre actualizada:

Hay que ir como va el mundo, al ritmo del mundo. Ahora es la informática. Antes no me llamaba la atención y prefería comunicarme por teléfono. Ahora estoy muy interesada en conocer sobre el tema, colaborar con las tareas de los niños. Ayer mi niño tenía un deber sobre el tema de Colombia, y para mí fue muy fácil entrar [en Internet] a ver todo lo referente a Colombia. [En casa] tengo ordenador pero no Internet (...) él ya sabe, pero a veces los muchachos quieren hacerle la trampita a uno [sic] de que sí fui y no encontré.

Feminismo/s 14, diciembre 2009, pp. 143-162 
En muchos testimonios, la principal motivación para informatizarse, para aprender y/o recordar, es la comunicación con hij@s que están geográficamente lejos. María, boliviana de 51 años, cuenta con orgullo que tiene dos hijas en Bolivia y un hijo en Buenos Aires. Antes de venir a Granada, hace menos de un año, él le enseñó a comunicarse por Internet con quienes quedaron en Bolivia. Si bien su hermana, que también vive en Granada, tiene un ordenador, ella prefiere revisar el correo desde el locutorio o desde las máquinas que usan en los cursos porque «si no es tuya, entonces da un poco de miedo, haces un click en cualquier sitio y se dispara para todos lados».

«Mis niños me decían: Ay mamá tenés que aprender, contó Laura, boliviana de 56 años, «y yo nunca daba importancia; como trabajaba, no me daba tiempo para nada. Y ahora, aquí, me arrepiento que no aprendí». Antes de viajar, hace cinco años, sus hijos la ayudaron a buscar información en Internet sobre los trámites administrativos necesarios para vivir y trabajar en España. Ahora lo que más ansía es aprender a chatear con ellos, pero, en Granada, dice que no puede porque trabaja a tiempo completo como empleada doméstica interna en la casa y el comercio de sus empleadores.

\subsection{El tiempo}

La carrera contra el tiempo es una experiencia bien conocida por mujeres de diversos perfiles socioeconómicos, étnicos y etarios. También fue un tema recurrente en las entrevistas, especialmente en los testimonios de las empleadas domésticas, para las que Internet no forma parte de su trabajo sino que se inscribe en el tiempo libre de ocio. Muchas de las mujeres entrevistadas, aunque tienen largas jornadas laborales, están lejos de sus familias y ello les deja más tiempo para realizar actividades que antes no concebían en sus apretadas agendas. En esos espacios, los cursos de Internet e informática encuentran su lugar. Así, Úrsula dice que quiere aprovechar para hacer los cursos ahora porque en Bolivia no le va a quedar «tiempo para estudiar ni nada». Socorro, nicaragüense de 54 años, expresó: «en mi país he trabajado todo el tiempo, no tenía tiempo ni para mi, y aquí teniendo la oportunidad, lo aprovecho». Ella también aspira a ser una mamá conectada, y su principal ambición es comunicarse con su hijo que vive en EE.UU. «Tengo casilla de correo porque me lo hizo mi amiga, entonces yo escribo y ella me dice cierre, abra y yo pongo la contraseña (...) Quiero aprender a hacerlo sola».

Quienes viven en Granada con su familia, tienen que negociar los tiempos y encontrar los intersticios libres. Regina suele tener «su media hora» de Internet por la noche y no más: «(...) salgo de mi trabajo, después a comer, después a hacer la compra, después a hacer la cena, y cuando quieres acordar 
el tiempo se voló». El tiempo en general también se les vuela a las hermanas peruanas de la Asociación de mujeres inmigrantes uno=uno, Susana y Jacinta. Susana de 52 años hace 16 que vive en Granada con su esposo, ambos profesionales. Si bien revisa su e-mail a diario desde el trabajo, dice que por las tareas del hogar no tiene tiempo desde su casa. Su hermana Jacinta, de 51 años, vino para mejorar su situación laboral. Desde que llegó a Granada en el 2005, trabaja como empleada doméstica interna cuidando a una señora mayor y tiene libres las tardes de 18:00 a 21:30 hs. En un centro de formación y empleo del Ayuntamiento, le informaron sobre cursos de capacitación a distancia por Internet, una solución de formación compatible con su horario de trabajo. Ya sabía algo de manejo de ordenadores por cursos que hizo estando en Perú, pero lo de Internet fue en Granada, un poco probando sola y otro poco con la ayuda de su hermana Susana:

Allá [en Perú] no tenía tiempo porque estaba muy liada entre mi trabajo, mi casa y mi familia. No me daba tiempo de meterme en Internet ni para averiguar cosas, en cambio aquí sí. Yo me decía lo haré un sábado o un domingo pero ya no me quedaba tiempo: el sábado a la lavadora, el domingo a la planchadera [sic], y ya el lunes otra vez. Y cuando tenía el tiempo para poder ir, ya estaba cansada, ya no me apetecía ir, me desganaba.

Jacinta tiene dos hijos adolescentes, uno en Perú y otro viviendo con ella. Cuenta cómo corría, literalmente, de su hogar como interna a un sitio con ordenadores y conexión para hacer los cursos, a su propia casa donde estaba su hijo solo. Los fines de semana el chico cocinaba y ella podía dedicar las cinco horas diarias que, por ejemplo, el curso de inglés online demandaba. Cuando conversamos estaba cursando auxiliar de enfermería y hacía pocas semanas que había llegado su marido de Perú:

Últimamente he dejado un poquito [el curso] porque está mi esposo recién llegado. Si él estuviera desde hace tiempo no pasaría nada porque sé que se puede manejar solo (...) Me da pena ponerme a estudiar y que está mi esposo ahí sentado. Entonces he dejado un poco eso. No me gusta, pero ¿qué hago? también tengo que darle tiempo a mi esposo.

\section{Consideraciones finales y parciales}

Este trabajo constituye un esfuerzo por aprehender, aunque sea parcialmente, el rico entramado de experiencias y universos de sentido que las personas construyen en relación a Internet en sus situaciones particulares, dotando de cuerpo y voz a las cifras de las estadísticas sobre usuari@s de la red. Mi propuesta específica ha sido atender a cómo un grupo de mujeres latinas inmigrantes residentes en Granada adoptan Internet en el espacio de la diáspora. 
Sus testimonios sugieren que las dinámicas de sus proyectos migratorios les han motivado a incursionar en Internet, superando barreras personales y estructurales que las situaban, consciente o inconscientemente, como ajenas a la CMC. Esta condición, que hemos llamado otredad digital, evidencia los mecanismos y discursos que otrorizan a las entrevistadas en su triple condición de mujeres, latinas inmigrantes y usuarias no avanzadas de Internet, en relación a un usuario tipo universal imaginado en hombres, blancos, educados y occidentales. Cabe destacar cómo esta otredad se filtra capilarmente en conceptos tales como brecha digital, de amplia circulación política, académica y mediática, y cuán necesarias son su deconstrucción y problematización. Pero, además de criticar esta tendencia, la propuesta de este artículo es rescatar las posibilidades empoderadoras de lo no categorizable por los discursos hegemónicos patriarcales y tecnodeterministas. En la misma línea que la «otredad inapropiada/ble» de Haraway y Minh-ha, se sugiere pensar en una otredad digital táctica, que aspira o ofrecer un lugar analítico para habitar por los cuerpos excluidos de tal modelo universal, de una manera productiva y afirmativa que atienda a las múltiples dimensiones y dinamismo de las diferencias, y cómo ello repercute en la creación de diversos e igualmente válidos repertorios de negociación y apropiación de Internet. En el espacio de la diáspora, las mujeres entrevistadas han experimentado cómo una necesidad práctica (trabajo, comunicación, formación) ha repercutido en pequeños grandes logros al nivel de sus subjetividades, en términos de autoestima, realización personal y la imaginación de nuevas posibilidades. En sus expresiones se desarrolla un ejercicio autopoiético de crearse como agentes legítimas en informática e Internet y de sus palabras emergen, más o menos explícitos, los procesos dinámicos de su continua construcción como sujetas. Han desafiado la imagen del usuario tipo de Internet en términos de género, edad, clase y etnia y, al proyectarse como usuarias, han superado sus propias barreras personales, miedos e inseguridades, revalorizando el tiempo propio y sus capacidades cognitivas.

Este trabajo es una contribución a los debates contemporáneos sobre Internet, desde la perspectiva que enfatiza la necesidad de considerar la tecnología no como un fin o un bien en sí mismo, sino como procesos que construyen y son construidos por las personas y grupos sociales. Ello podría contrarrestar, o al menos matizar, los discursos tecno-optimistas sobre el potencial democratizador y revolucionario de Internet, y los discursos tecno-pesimistas que deslegitiman las posibilidades de diferentes personas o grupos para apropiarse de la tecnología y adaptarla a sus propias necesidades. 


\section{Bibliografía}

Appadurai, Arjun. «Here and now» en N. MirzoefF (ed) The Visual Culture Reader, Londres, Routledge, 2002, pp. 173-179.

BAKARDJIEVA, Maria. Internet society: The internet in everyday life. Londres, Sage, 2005.

BRAH, Avtar. «Diaspora, Border and Transnational Identities». En R. LEWIS y S. Mills (eds.). Feminist Postcolonial Theory: A Reader. Edimburgo, Edinburgh University Press, 2003, pp. 613-634.

CASTAÑo, Cecilia (ed). La segunda brecha digital. Madrid, Cátedra, 2008.

CASTELLS, Manuel. "Materials for an exploratory theory of the network society". British Journal of Sociology, vol. 51, no 1 (2000), pp. 5-24.

DirLIK, Arif. «The Global in the Local': The Postcolonial Aura. Third World Criticism in the Age of Global Capitalism». En A. McClintock y A. Mufti (eds) Dangerous Liaisons. Gender, Nation and Postcolonial Perspectives. Minneapolis, University of Minnesota Press, 1997, pp. 501-528.

Fernández, María. «Postcolonial Media Theory». Art Journal, vol. 58, no 3 (1999), pp. 58-73.

FrisSEN, Valerie. "The myth of the digital divide». En A. PiCOT, J.C. BurgELman y K. SCHRAPE. E-merging media: Communication and the media economy of the future, European Communication Council Report. Berlin, Springer, 2005, pp. 271-284.

GurumurThy, Anita. Gender and ICT. Overview report. Institute of Development Studies, $2004<$ http://www.bridge.ids.ac.uk/reports_gend_CEP.html\#icts>, consultado el 19-05-2009.

Haraway, Donna. «The Promises of Monsters: A Regenerative Politics for Inappropriate/d Others». En L. Grossberg, C. Nelson y P. A. Treichler (ed). Cultural Studies. Nueva York, Routledge, 1992, p. 295-337.

- Testigo_modesto@segundo_milenio_Hombrehembra_conoce Oncoratón. Nueva York, Routledge, 1997.

- The Haraway Reader. Nueva York, Routledge, 2004.

HiLler, Harry H. y Franz, Tara M. «New ties, old ties and lost ties: the use of the internet in diaspora». New Media and Society, vol. 6, nº 6 (2004), pp. 731-752.

LEANDER, Keavin. «Locating Latanya: The Situated Production of Identity Artifacts in Classroom Interaction». Research in the Teaching of English, vol. 37, $\mathrm{n}^{\circ}$ 2 (2002), pp.198-250.

LEDWITH, Valerie et al. «Immigration, the internet, and spaces of politics». Political Geography, no 21 (2002), pp. 989-1012.

LIE, Merete. «The new Amazons. Gender symbolism on the Net». En M. LIE (ed.). He, She and IT Revisited: New Perspectives on Gender in the Information Society. Oslo, Gyllendal Norsk Forlag, 2003, pp. 251-276. 
Lievrouw, Leah A. y LivingSTONE, Sonia M. «Introduction». En L.A. Lievrouw y S.M. Livingstone (eds). Handbook of New Media. Social Shaping and Consequences of ICTs. Londres, Sage, 2002, pp.1-16.

Lievrouw, Leah A. «Determination and Contingency in New Media Development: Diffussion of Innovations and Social Shaping of Technology Perspectives» en L.A. Lievrouw, y Livingstone, S. (eds.) Handbook of New Media. Social Shaping and Consequences of ICTs. Londres, Sage, 2002, p. 183-200.

LIVINGSTONE, Sonia M. «Part one: the changing social landscape. Introduction» en L. A. Lievrouw. y S.M. Livingstone (eds.). Handbook of New Media. Social Shaping and Consequences of ICTs. Londres, Sage, 2002, pp. 17-21.

LYKKE, Nina. «Feminist Cultural Studies of Technoscience and Other Cyborg Studies. A Cartography». En R. BRAIDOTTI et. al. The Making of European Women's Studies Volume IV, Utrecht, Athna, 2002, pp. 133-143.

MAHAN, Amy. «ICT indicators for advocacy». En Global Information Society Watch 2007 Report. Montevideo, APC e IteM, 2007, pp. 77-87.

MitrA, Ananda. «Marginal Voices in Cyberspace». New Media Society, vol. 3, n 1 (2001), pp. 29-48.

- «Theorizing Cyberspace: the idea of voice applied to the Internet discourse». New Media Society, vol 4, no 4 (2005), pp. 479-498.

Morales, Juan J. y RodríGueZ, $M^{\mathrm{a}}$ del Carmen. «Inmigración y ciudad digital: reflexiones entorno a la tercera brecha digital», Archivo del Observatorio para la CiberSociedad, 2008. <http://www.cibersociedad.net/archivo/articulo. php? art = 232> consultado el 05-03-2009.

Mosco, Vincent. The Digital Sublime. Myth, Power, and Cyberspace. Massachusetts, MIT Press, 2004, pp. 17-49.

PAASONEN, Susanna. «Free Tourists in the Land of Plenty? From cyberdiscourse towards politics of location», en Communication Front 2000 Book, Crossing Points East-West, 2000. <http://www.cfront.org/cfoobook/en/susanna-locationen>, consultado el 05-03-2009.

PISCITELLI, Alejandro. «Inmigrantes digitales vs. nativos digitales. La migración digital, un concepto bastante ambicioso, sin fecha <http://portal.educ.ar/ debates/educacionytic/nuevos-alfabetismos/inmigrantes-digitales-vs-nativosdigitales.php>, consultado el 22-06-2009.

POSTER, Mark. What's the matter with the Internet?. Minneapolis, University of Minnesota Press, 2001.

Robertson, Roland. Globalization. Social Theory and Global Culture. Londres, Sage, 1992.

Silverstone, Roger. Media and Technology in the Everyday Life of European Societies. Informe del European Media and Technology in Everyday Life Network, 2000-2003. London School of Economics and Political Science, 2003. 
<http://www.lse.ac.uk/collections/EMTEL/reports/silverstone_2003_emtel. pdf>, consultado el 20-10-2009.

MarTínez, Manuel; Tucho, Fernando y García de MAdARIAGA, José Mª . «emocracia Digital: Nuevos Medios y Participación Ciudadana. Experiencias en la Red de la Población Inmigrante en España». Portuaria, vol. 5, nº 2 (2005), pp. 21-34.

VAN Dijk, Jan y HACKER, Kenneth. «The Digital Divide as a Complex and Dynamic Phenomenon». The Information Society, no 19 (2003), pp. 315-326.

VAN ZoOnEn, Liesbet. «Feminist Internet Studies». Feminist Media Studies, vol. 1, $n^{\circ} 1$ (2001), pp. 67-72.

VehVILÄINEN, Marja. «Understandings of Gender and Information Technology». En C. MÖrtBerg (ed.). Where do we go from here? Feminist Challenges to Information Technology. Luleå University of Technology, 2000, pp.17-38.

- «Gendered agency in Information Society: on located politics of technology». En S. Paasonen y M. Consalvo (eds.). Women and Everyday Uses of the Internet: Agency and Identity. Nueva York, Peter Lang Publishing, 2002, pp. 274-288.

WEBSTER, Frank. «The Information Society Revisited». En L. LIEVROUW. y S. LIVInGStOnE (eds.). Handbook of New Media. Social Shaping and Consequences of ICTs. Londres, Sage, 2002, pp. 22-33. 